\title{
Mobile VoIP User Experience in LTE
}

\author{
Karl Andersson, Seraj Al Mahmud Mostafa, and Raihan UI-Islam \\ Pervasive and Mobile Computing Laboratory \\ Department of Computer Science, Electrical and Space Engineering \\ Luleå University of Technology \\ SE-971 87 Luleå, Sweden \\ \{karl.andersson@,sermos-9@student.,raiuli-0@student.\}ltu.se
}

\begin{abstract}
GPP Long-term Evolution (LTE) systems being deployed are fast gaining market shares. High data rates (approaching $100 \mathrm{Mbit} / \mathrm{s}$ in the downlink direction and $50 \mathrm{Mbit} / \mathrm{s}$ for uplink connections) and small delays are attractive features of LTE. Spectrum flexibility also makes deployment easy on various frequency bands in different parts of the world. However, as LTE offers packet switched services only, mobile broadband connectivity has become the dominant LTE application so far. This paper studies user-perceived quality of service for a mobile Voice over IP (VoIP) application in LTE. Results were achieved using the OPNET Modeler simulation environment.
\end{abstract}

Keywords-LTE; VoIP; User-perceived quality of service

\section{INTRODUCTION}

3GPP Long-term Evolution (LTE) [1] is the latest standard in the GSM/UMTS line specified in 3GPP Release 8. It replaces the WCDMA transmission scheme of UMTS so that OFDMA (Orthogonal Frequency-Division Multiple Access) is used for downlink while SC-FDMA (Single-carrier FDMA) is used for uplink traffic.

Orthogonal frequency-division multiplexing (OFDM) is an FDM type of scheme that is used as a digital multi-carrier modulation method where a number of closely spaced orthogonal sub-carriers are used to carry data. The data is divided into several parallel data streams or channels, one for each sub-carrier. A flexible resource allocation is achieved through dynamic assignment of sub-carriers to a specific node.

Each sub-carrier is modulated with a conventional modulation scheme at a low symbol rate. Furthermore, MIMO (multiple-input, multiple-output) antenna technology is used in LTE. Minimum transmission time interval (TTI) is $1 \mathrm{~ms}$ and 64QAM was added as a modulation scheme.

The Dedicated Traffic Channel (DTCH) in LTE is mapped to DLSCH and ULSCH (Downlink Shared Channel and Uplink Shared Channel) respectively. It uses HARQ and adapts dynamically to the link quality. Spectrum flexibility was an important design goal for LTE and it was built to scale using bandwidths ranging from $1.4 \mathrm{MHz}$ to $20 \mathrm{MHz}$ in both paired and unpaired configurations. A wide range of frequency bands are expected to be used for LTE including the $700 \mathrm{MHz}$ band allowing for indoor usage and wide coverage.

LTE provides data rates up to $100 \mathrm{Mbits} / \mathrm{s}$ in the downlink direction, uplink data rates up to $50 \mathrm{Mbps}$ in the uplink direction and latencies in the radio access network at 10 milliseconds. The system is non-backward compatible with GSM or UMTS and hence requires a new infrastructure. The upgraded version LTE Advanced is designed to meet the requirements from the fourth generation (4G) radio access network of $1 \mathrm{Gbits} / \mathrm{s}$ in data rate for stationary applications and $100 \mathrm{Mbits} / \mathrm{s}$ for mobile applications. The first commercial LTE network was opened in Stockholm and Oslo in December 2009. A wide range of frequencies are expected to be used.

The structure of LTE networks is changed radically from the GSM and UMTS network structures, see figure 1. eNB (Evolved NodeB) is the only node type in EUTRAN (Evolved UTRAN) responsible for all radio interface-related functions. Main node types in the EPC (Evolved Packet Core) are the MME (Mobility Management Entity) responsible for mobility, UE (User Equipment) identity, and security management functions, the S-GW (Serving Gateway) terminating the interface towards E-UTRAN, and the PGW (PDN Gateway) terminating the interface towards the packet data network (PDN).

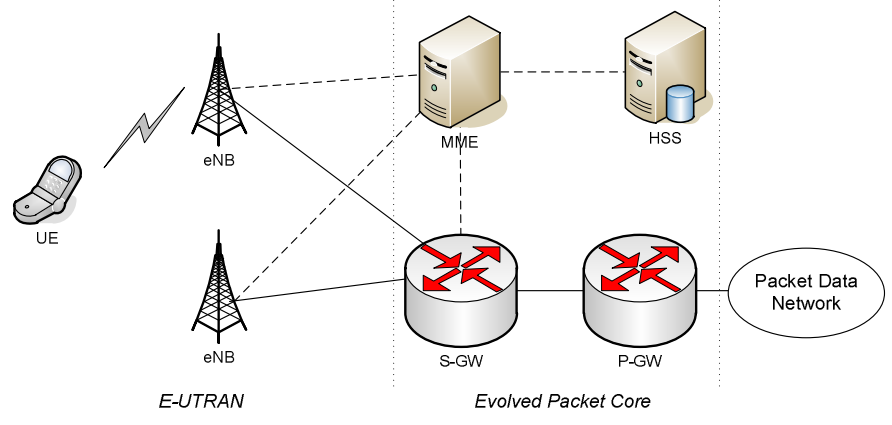

Figure 1. LTE network structure

It should be noted that the circuit switched domain was finally removed from the network architecture. In LTE voice services are not delivered through dedicated nodes in the core network, but through VoIP-based mechanisms in other subsystems like Multimedia Telephony (MMTel) [2] in the IP Multimedia Subsystem [3]. Also, 3GPP decided to define a circuit switched fall back (CS fallback) approach [4] as an intermediate solution. Furthermore, 3GPP also began to study yet another approach, namely circuit-switched over packetswitched system (CS over PS). That work was taken over by the VoLGA Forum [5] working on support of voice services over LTE using the GAN (Generic Access Network) standard. 


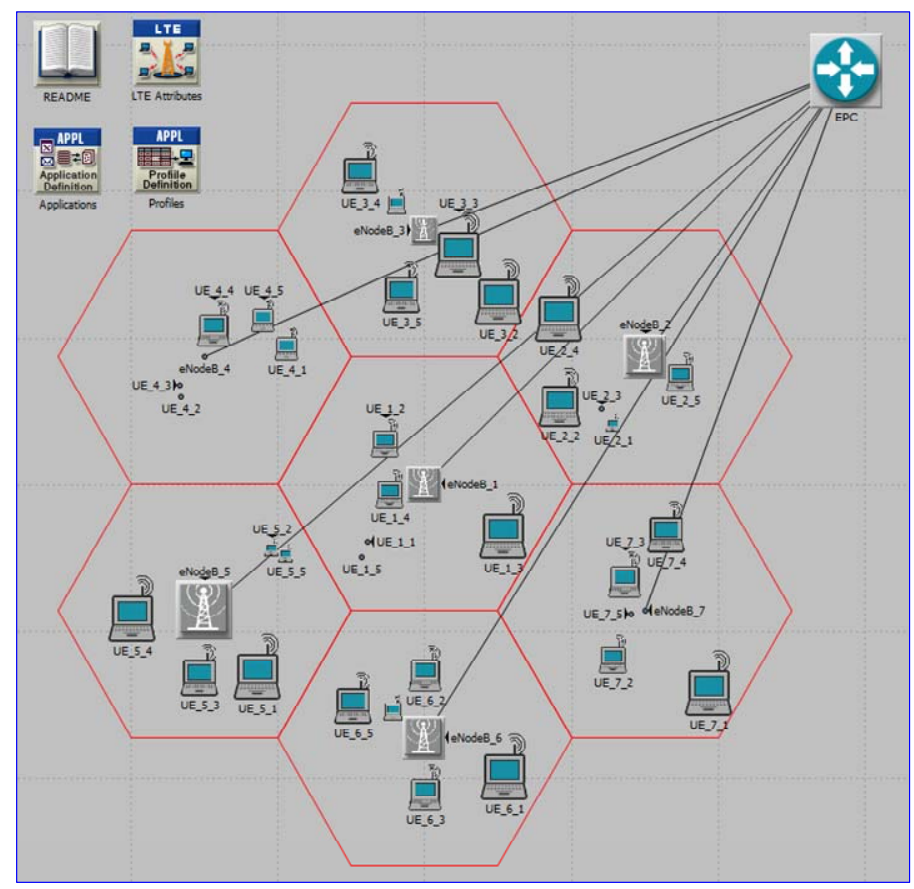

Figure 2. Evaluation setup

Trying to get back to the initial idea of using IMS for voice services in LTE, the "One Voice" initiative was launched. That work then became the basis of the GSMA's "Voice over LTE" (VoLTE) [6] initiative.

This paper presents a comparative study on user-perceived quality of service for Mobile VoIP applications in LTE networks. Results are presented as user-perceived quality of service in terms of Mean Opinion Score (MOS) values for various system bandwidths and codec types.

The rest of the paper is organized the following way: Section II surveys related work. Section III presents the evaluation framework and simulations performed. Section IV presents results achieved, while Section V finally concludes the paper and indicates future work.

\section{RELATED WORK}

Gavrilovic [7] surveyed standard solutions for voice and SMS services in LTE networks. IMS Telephony (MMTel), IMS Telephony with handover to CS domain (SRVCC), and CS fallback were qualitatively compared. The author concluded MMTel creates possibilities for new service offerings like video calls, chat, and image sharing, whereas SRVCC handles the problem of initial spotted LTE coverage well. Finally, Gavrilovic concluded CS fallback features enables operators to decouple the LTE rollout from IMS/MMTel rollout.

Paisal [8] also qualitatively compared various techniques for delivering VoIP services in LTE networks including CS Fallback, IMS Telephony with handover to CS domain (SRVCC), and Voice over LTE via Generic Access (VoLGA). Paisal concluded CS Fallback and/or VoLGA would be of most interest to operators not intending to deploy IMS. Operators having $2 \mathrm{G} / 3 \mathrm{G}$ networks and planning to deploy IMS could consider CS fallback or VoLGA as an intermediate step, but use SRVCC later when IMS has been installed. Those operators deploying IMS together with LTE should go for SRVCC directly, Paisal argued.

Puttonen et al. [9] quantitatively studied VoIP performance in LTE downlink (DL) using the Adaptive Multi-Rate (AMR) [10] 12.2 codec in four simulation cases standardized by 3GPP. In particular the effects of link adaptation, packet bundling, control channel capacity, and number of Hybrid ARQ (HARQ) processes on VoIP capacity were studied. Maximum capacity was reported to be 60 and 300 UEs per cell at $1.25 \mathrm{MHz}$ and 5 $\mathrm{MHz}$ system bandwidth respectively. It was also reported that link adaptation together with packet bundling improves the overall VoIP capacity in an LTE network. Finally, it was concluded in the paper that control channel limitations can be compensated by packet bundling.

Henttonen et al. [11] studied the effects of using robust header compression (ROHC) techniques in LTE networks in highly mobile scenarios for VoIP applications. Capacity loss from highly mobile $(120 \mathrm{~km} / \mathrm{h})$ scenarios was reported to reach $65 \%$ compared to near-stationary $(3 \mathrm{~km} / \mathrm{h})$ scenarios. The authors also reported that the overall capacity loss of using non-ideal ROHC compared to using ideal $\mathrm{ROHC}$ reached 1$7 \%$.

Our previous work includes a study on user-perceived quality of service for multimedia applications in wireless heterogeneous networks [12]. A software prototype was built and experiments were performed in a combined WLAN/CDMA2000 heterogeneous network. Furthermore, a study on session setup delay and jitter for a Voice over WLAN (VoWLAN) application was done using composite metrics [13]. Also, a model optimizing multimedia quality of experience (QoE) using prediction and statistical learning was 
TABLE I. SimULATION SETtings

\begin{tabular}{|c|c|}
\hline Parameter Description & Parameter Value(s) \\
\hline System bandwidths & $\{1.4,20\} \mathrm{MHz}$ \\
\hline VoIP codecs & $\{$ G.711, G.723.1 5.3K, G.729 A, GSM FR $\}$ \\
\hline Duplex mode & FDD \\
\hline Base frequency (UL) & $1920 \mathrm{MHz}$ \\
\hline Base frequency (DL) & $2110 \mathrm{MHz}$ \\
\hline Cyclic prefix type & 7 symbols per slot \\
\hline
\end{tabular}

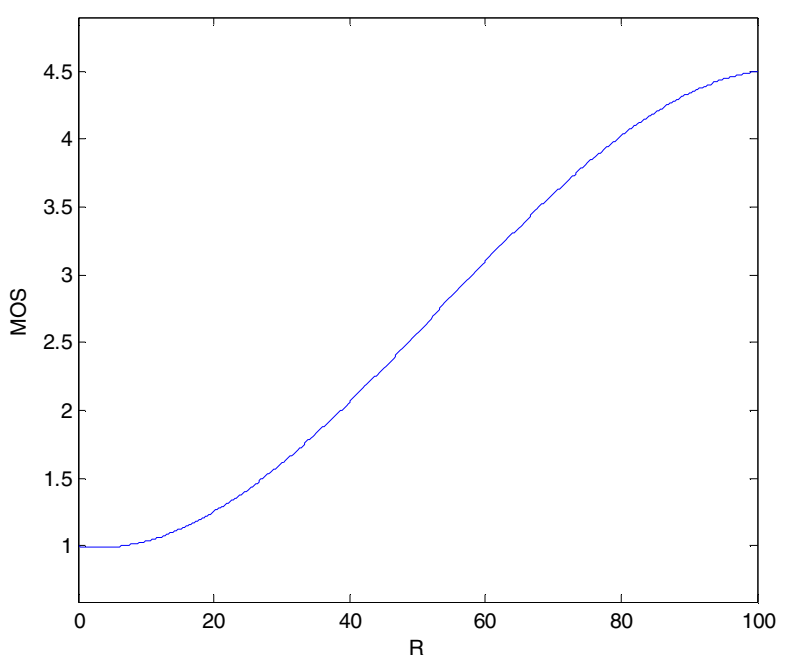

Figure 3. Relationship between MOS values and R values

proposed [14]. Finally, an access network selection mechanism for mobile nodes in a combined WLAN/LTE environment was proposed and evaluated [15]. VoIP performance in terms of user-perceived quality of service was the main parameter being optimized.

\section{EVALUATION FRAMEWORK AND SIMULATIONS}

In order to study user-perceived quality of service for VoIP applications in LTE networks, simulations using the OPNET Modeler [16] simulation environment were undertaken. The evaluation setup is depicted in figure 2 .

Seven cells with five UEs per cell were configured, so that each UE started a phone VoIP call to any other randomly selected UE. Simulation settings are found in Table 1.

MOS values were used as the studied metric. Such values can be calculated using the E-model [17] according to the following formulae:

$$
\begin{gathered}
R=R_{o}-I_{s}-I_{\mathrm{d}}-I_{e-e f f} \\
I_{e-e f f}=I_{e}+\left(95-I_{e}\right) \cdot \frac{P p l}{\text { Ppl/BurstR+Bpl }}
\end{gathered}
$$

$$
\text { MOS }=\left\{\begin{array}{cc}
1, & \text { for } R<0 \\
1+0.035 \cdot R+R \cdot(R-60) & \text { for } R \in[0,100] \\
\cdot(100-R) \cdot 7 \cdot 10^{-6}, & \text { for } R>100 . \\
4.5 &
\end{array}\right.
$$

TABLE II. MOS VALUES AND THEIR INTERPRETATION

\begin{tabular}{|c|c|}
\hline MOS Value & Impairment \\
\hline 5 & Imperceptible \\
\hline 4 & Perceptible, not annoying \\
\hline 3 & Slightly annoying \\
\hline 2 & Annoying \\
\hline 1 & Very annoying \\
\hline
\end{tabular}

TABLE III. Simulation Results (MOS VAlues)

\begin{tabular}{|c|c|c|}
\hline \multirow{2}{*}{ VoIP Codec } & \multicolumn{2}{|c|}{ System Bandwidth } \\
\cline { 2 - 3 } & $\mathbf{1 . 4} \mathbf{M H z}$ & $\mathbf{2 0} \mathbf{M H z}$ \\
\hline GSM FR & 2.51 & 3.49 \\
\hline G.729 A & 3.02 & 3.03 \\
\hline G.723.1 5.3K & 2.51 & 2.51 \\
\hline G.711 & 3.64 & 3.64 \\
\hline
\end{tabular}

$R_{\mathrm{o}}$ is the basic signal-to-noise ratio defined as 93.2 [18], $I_{s}$ represents impairments to the source signal, and $I_{d}$ is the impairment due to delay and echo effects. $I_{e-e f f}$ represents impairments due to packet losses.

Furthermore, $P p l$ represents instantaneous packet loss rate, while BurstR is the mean number of packets lost in a burst of lost packets at each second. $\mathrm{Bpl}$ is the robustness factor to random packet loss and was set to $B p l=20$ for the GSM Fullrate (FR) codec [17].

Figure 3 depicts the relationship between MOS values and $R$ values, while interpretation of MOS values can be found in Table II.

\section{RESULTS}

After simulating VoIP traffic in the LTE network, we got results according to Table III.

Obviously, using high bitrate codecs (the G.711 with a nominal bitrate of $64 \mathrm{~kb} / \mathrm{s}$ ) gives higher MOS values. The other more low-bitrate codecs (GSM FR, G.723.1 5.3K, and G.729 A) give lower MOS values. The GSM FR codec, however, performs quite well in the $20 \mathrm{MHz}$ system bandwidth scenario.

\section{CONCLUSIONS AND FUtURE WORK}

Voice services in LTE are still being defined and standardized. Today, mobile broadband is the predominant service delivered in commercial LTE networks. Voice being a very important feature in all telecommunication networks and also the main revenue source for operators makes standardization of voice services in LTE a very important issue.

Our simulations show a variety of codecs may be used for delivering voice services with good enough quality in LTE networks.

We intend to continue this work and add different mobility scenarios to the simulation models and perform an extended study. Also, we plan to do live experiments with a few commercial LTE networks to see how our simulated results compare with experimental results.

\section{REFERENCES}

[1] 3GPP TS 36.401, Evolved Universal Terrestrial Radio Access (EUTRA) and Evolved Universal Terrestrial Radio Access Network (EUTRAN) Overall description, Stage 2. June 2010. 
[2] 3GPP TS 22.173, IP Multimedia Core Network Subsystem (IMS) Multimedia Telephony Service and supplementary services, Stage 1. September 2009.

[3] 3GPP TS 23.228, IP Multimedia Subsystem (IMS), Stage 2. March 2010.

[4] 3GPP TS 23.272, Circuit Switched (CS) fallback in Evolved Packet System (EPS), Stage 2. June 2011.

[5] VoLGA Forum. Available at www.volga-forum.com. Accessed on August 4, 2011.

[6] GSMA, Voice over LTE. Available at www.gsmworld.com/ourwork/mobile_broadband/VoLTE.htm. Accessed on August 4, 2011.

[7] S. Gavrilovic. Standard Based Solutions for Voice and SMS Services over LTE. In Proceedings of the 33rd International Convention (MIPRO 2010), Opatija, Croatia, May $24-28,2010$.

[8] V. Paisal. Seamless Voice over LTE. In Proceedings of 4th IEEE Conference on Internet Multimedia Services Architecture and Application (IMSAA 2010), Bangalore, India, December 15-17, 2010.

[9] J. Puttonen, T. Henttonen, N. Kolehmainen, K. Aschan, M. Moisio, and P. Kela. Voice-Over-IP Performance in UTRA Long Term Evolution Downlink. In Proceedings of the 2008 IEEE 67th Vehicular Technology Conference (VTC-Spring 2008), Singapore, May 11-14, 2008.

[10] 3GPP TS 26.093, Mandatory speech codec speech processing functions, Adaptive Multi-Rate (AMR) speech codec, Source controlled rate operation, Release 10. March 2011.

[11] T. Henttonen, K. Aschan, J. Puttonen, N. Kolehmainen, P. Kela, M. Moisio, and J. Ojala. Performance of VoIP with Mobility in UTRA Long
Term Evolution. In Proceedings of the 2008 IEEE 67th Vehicular Technology Conference (VTC-Spring 2008), Singapore, May 11-14, 2008.

[12] K. Andersson, D. Granlund, and C. Åhlund. $M^{4}$ : Multimedia mobility manager: a seamless mobility management architecture supporting multimedia applications. In Proceedings of the 6th International Conference on Mobile and Ubiquitous Multimedia (MUM '07), Oulu, Finland, December 12-14, 2007.

[13] M. Elkotob and K. Andersson. Analysis and measurement of session setup delay and jitter in VoWLAN using composite metrics. In Proceedings of the 7th International Conference on Mobile and Ubiquitous Multimedia (MUM '08), Umeå, Sweden, December 3-5, 2008.

[14] M. Elkotob, D. Granlund, K. Andersson, and C. Åhlund. Multimedia QoE Optimized Management Using Prediction and Statistical Learning. In Proceedings of the 35th IEEE Conference on Local Computer Networks (LCN 2010), Denver, CO, USA, October 10-14, 2010.

[15] K. Andersson and C. Åhlund. Optimized Access Network Selection in a Combined WLAN/LTE Environment. To appear in Wireless Personal Communications, Fall 2011.

[16] OPNET Modeler 16.0.A, PL6

[17] The E-model, a Computational Model for Use in Transmission Planning. ITU Recommendation G.107, April 2009

[18] Transmission Impairments due to Speech Processing. ITU Recommendation G.113, November 2007 